\title{
Studies on the Genetic Multiplicity of a Gene in Yeast Cells, I Characteristics of the Induced Mutations by Subcritical Temperature and Ultraviolet Light ${ }^{1)}$
}

\author{
Takashi Ito, T. Yamasaki and Y. Matsudaira \\ Biophysical Laboratory, Department of Physics, St. Paul's (Rikkyo) University, Tokyo
}

Received March 9, 1962

\section{Introduction}

It seems well established by using DNA-attacking mutagenic agents that phage DNA behaves as if it has a duplex nature in transferring genetic information to the progeny (e.g. Freese et al. 1961). With higher organisms, however, such experimental evidence for the duplex nature of a so-called gene is very scarce. Only the experiments carried out by Kaudewitz (1959) and his associates (Kaudewitz et al. 1958) with $E$. coli treated with nitrous acid and $\mathrm{P}^{32}$ come to the authors' attention, where, except for $\mathrm{P}^{32}$, sectored colonies composed of auxotroph and prototroph were not found although the mutation induction was an event of two hit-type. Under these circumstances it may be useful to know the response of a gene to one-hit type and two-hit type agents ${ }^{2}$ and the present experiment was performed with genetically well-defined yeast cells to know whether a gene has duplex nature or not. The gene mutation at the ad locus was studied.

As mutagenic agents, subcritical temperature for one-hit type action and ultraviolet light for two-hit action were used. The results revealed remarkable difference in relative frequencies of whole-colony mutation and fractional-colony mutation indicating genetic duplex nature of the gene.

Very recently, Altenburg and Browning (1961) and Fahmy and Fahmy (1961) reported similar experiments in Drosophila, the former using X-rays and chemicals, and the latter chemicals.

\section{Material and Methods}

Material: Diploid yeast cells ( $\left.\alpha a d^{-} a r g^{-} h i s^{+} / a a d^{+} a r g^{-} h i s^{-}\right)$were prepared from

1) This work was supported by a grant from the Ministry of Education, and part of the work was presented orally by the senior author at the Annual Meeting of the Genetics Society of Japan, Sendai, Sept. 2, 1961.

2) We use the terms one-hit type action and two-hit type action in the following sense: when a single event is required to affect both strands of the DNA molecule, it is of the one-hit type action; and when two events are required to affect both strands, it is of the two-hit type action-irrespective of any difference in the way the action occurs. 
haploid stocks, $\alpha a d^{-} a r g^{-} h i s^{+}$and $a a d^{+} a r g^{-} h i s^{-}$, which were derived from Lindegren $8256 a \mathrm{ad}^{-}$and Lindegren $19861 \alpha \mathrm{arg}^{-} h \mathrm{si}^{-} l \mathrm{ys}^{-}$. It was assured by the screening on the minimal agar supplemented with arginine that only diploid cells heterozygous for adenine locus had been picked up. After cultivation in nutrient broth the cells were subjected to synchronization ( 3 hours incubation in liquid synthetic medium following 24 hours shaking in minimal medium at $30^{\circ} \mathrm{C}$ ). A culture in which at least $80 \%$ of the cell population was an early stage of budding was treated with the mutagenic agents.

Temperature treatment: For the temperature treatment, about $2 \mathrm{ml}$ of cell suspension $\left(10^{8 \sim 9}\right.$ cells $\left./ \mathrm{ml}\right)$ were placed in a small steel tube, and the tube was immersed in a $60^{\circ} \mathrm{C}$ water bath of large volume for various periods, 4 minutes in most cases. The tube was then cooled for 20 seconds in air, followed by 20 seconds in water $\left(25^{\circ} \mathrm{C}\right)$.

$U V$ treatment: The source of ultraviolet light was a germicidal lamp. The cells were irradiated after being spread on an agar plate. Doses were controlled to attain a certain fraction of survival, above $98 \%$, throughout the experiment.

Procedure: Samples for the treatment and for the control (for spontaneous origin) were taken as simultaneously as possible from a single culture in every set of experiments. In a series of experiments viable cells (colony forming centers) on nutrient agar were controlled to give around 400 colonies on each plate. (If the number of colonies exceeds 800 per plate, for example, the matter of disturbance by the resulting overlapping of the colonies becomes serious, especially for counting of fractionalcolony).

After the plates had been incubated for 2 days at $30^{\circ} \mathrm{C}$ induced variants were counted according to the classification of whole-colony mutation and fractional-colony (sectored colony) mutation, which were identified by phenotypic pink color of the colony attributable to the induced forward mutation on one of the homologue genes, $a d^{+} \rightarrow a d^{-}$(see Fig. 2). One reason for using diploid cells instead of haploid $a d^{+}$cells is simply because of the high viability of diploid cells to the treatment employed (particularly to high temperature). In other words, it is not probable that a haploid cell grows to fractional-colony, even if it has a relevant mutation on a strand, since any concurrent lethal damage (or lethal mutation) by the treatment would lead it to either non-mutant (white) colony formation or mutant (pink) colony formation depending on which strand was damaged. Diploid cell has much higher chance to survive in the same situation and would give a detectable frequency of fractional colony in a reasonable scale of experiment. Besides, in using diploid cells heterozygous for adenine locus, the advantages are that the spores from any cell in the formed colonies can be tested whenever desired; and that the results so obtained can be related to that locus.

Adenine requirement of both pink and white isolates from the induced variants was checked by plating them on minimal agar.

Precausion against photoreversion was not taken particularly in the present experiments. 


\section{Results}

Several possible changes induced by mutagenic agents and the resulting colony patterns are given in Fig. 1 under the assumptions of dual structure of the gene and of its semiconservative replication. In the last column of the same figure, the genetic characters of the spores which are to be obtained from the white portion of the sectored colony (white isolate) are indicated.

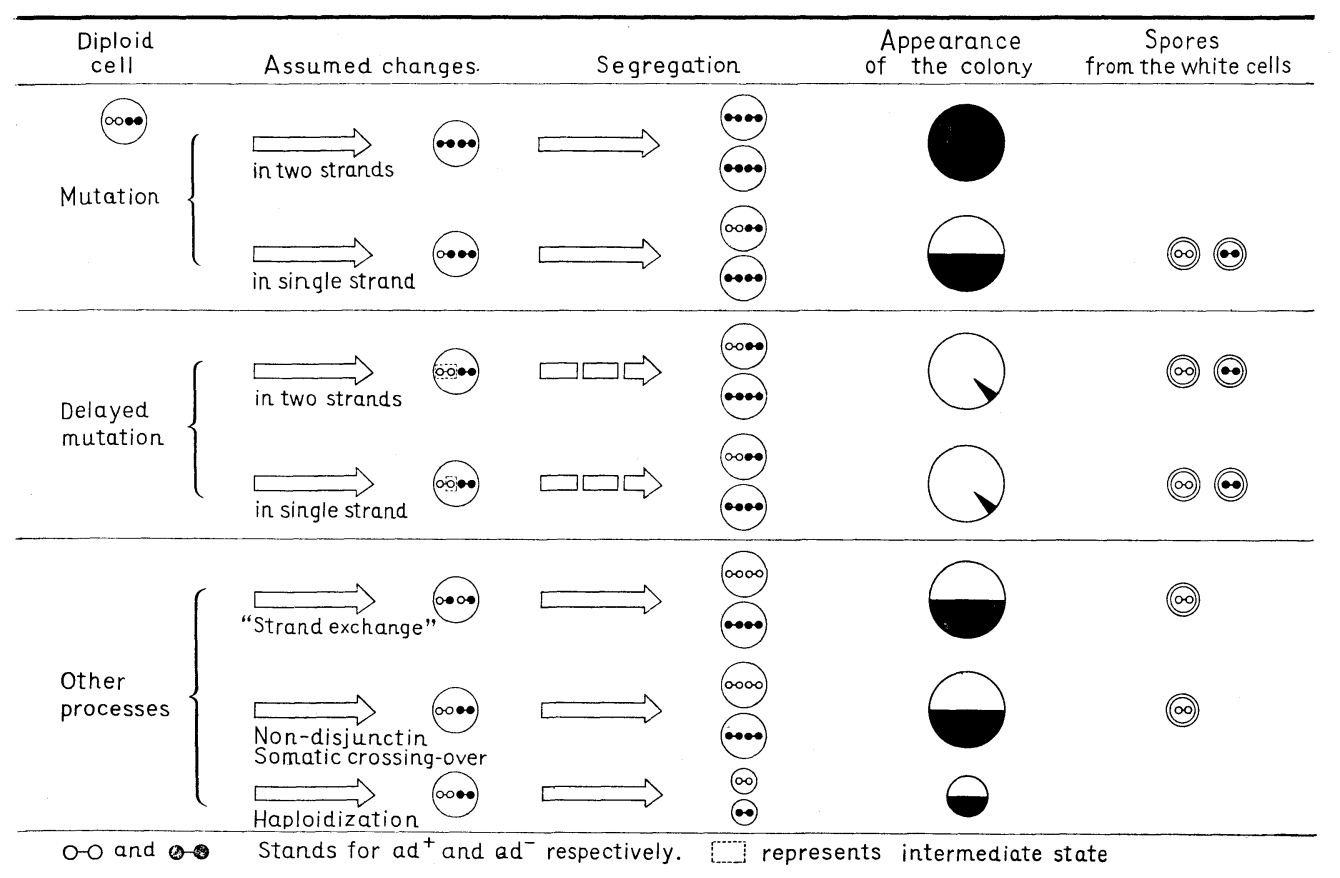

Fig. 1. Schemes of the possible changes in gene-DNA molecules of double helix by mutagenic agents, and the resulting colonies with some characters of the spores.

The kinds of mutant colonies are shown in Fig. 2. As for whole-colony mutation there is nothing unusual except for very rare occurrence of haploidization which has been detected by the microscopic observation of shape, size and ability of sporulation of the component cells. In fractional-colony mutation the trouble is that various degrees of sectors are observed (Fig. 2 b). This may probably be due to different growth rates of non-mutant cells and mutant cells in a colony, although other possibilities such as delayed mutation can not be ruled out. In fact, very small sectors or non-simple (irregular) sectors were occasionally observed in addition to the expected half-sectors (Fig. 2 a).

The results are shown in Table 1 . Here a remarkable difference in the relative frequencies of whole- and fractional-colony mutation between temperature treatment and UV treatment is apparent. Furthermore some portion of the mutations listed under "undefined" undoubtedly goes to whole-colony mutation since a considerable 
portion of the undefined may come from overlapping of non-mutant and wholly mutant colonies. So an explanation would be that by temperature, most of the mutations are changes induced in both strands of gene-DNA molecule whereas by UV, they are changes in one of the two strands.

In Fig. 3 the one-hit nature of temperature is further confirmed, since over a wide range of total mutation frequency the ratio of whole to total mutation remains unchanged. For the UV treatment, although data over a narrow range were obtainable, there is no evidence of one-hit nature.

Half sectors may be induced through either mutation or somatic recombination or both (see Fig. 1). To determine the contribution of somatic recombination we used a test in which the spores from the white isolate of the sectored colony were plated to see if they give only white colonies (somatic recombination) or both pink and white colonies (mutation). This test was performed for randomly sampled 10 sectored colonies induced by temperature and 21 sectored colonies induced by UV. Such test showed that 1 out of 10 was not heterozygous for the temperature induced and 7 out of 21 were not heterozygous for

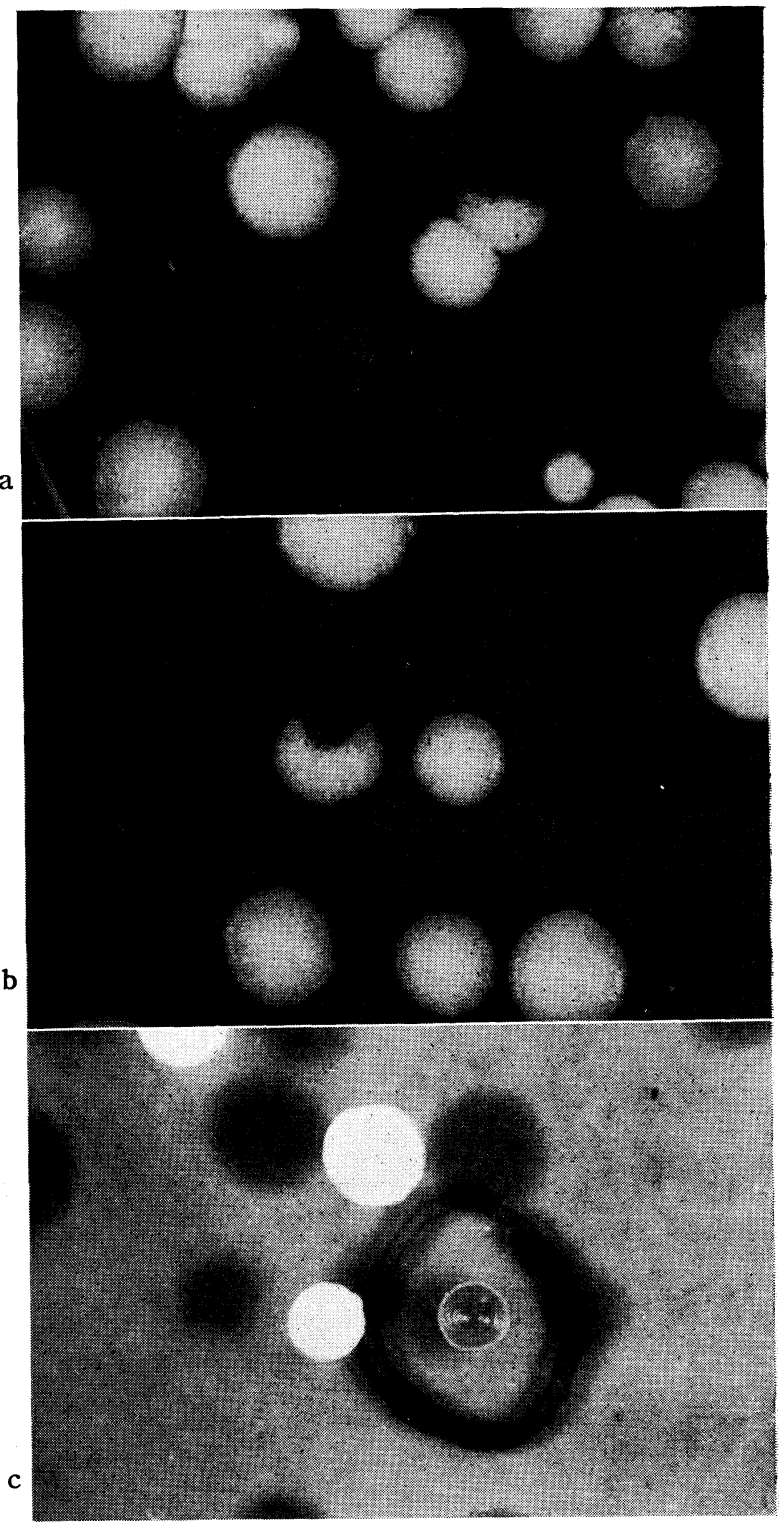

Fig. 2. Patterns of the colonies.

a, UV induced half-sector, typical fractionalcolony. b, Fractional-colony of less than half (UV induced). c, Whole-colony (temperature induced). the UV induced. So, especially in the UV treatment, somatic recombination may explain some $30 \%$ of the induced sectored colonies. However, for the remaining $70 \%$ of them we have to seek for a new mechanism. 
Table 1. Whole-colony mutations versus fractionals on ad locus in yeast cells (budding stage)

\begin{tabular}{|c|c|c|c|c|c|c|}
\hline Series & $\begin{array}{l}\text { No. colonies } \\
\text { counted }\end{array}$ & $\begin{array}{l}\text { Total } \\
\text { mutants }\end{array}$ & Fractional & Whole & Undefined & $\begin{array}{l}\text { Percent whole } \\
\text { (of total mut.) }\end{array}$ \\
\hline $\begin{array}{l}60^{\circ} \mathrm{C} 1.5 \mathrm{~min} ., 2.0 \mathrm{~min} . \& \\
4.0 \mathrm{~min} . \text { combined }\end{array}$ & 189,120 & 870 & 198 & 513 & 159 & 59 \\
\hline Ultraviolet & 20,018 & 69 & 50 & 10 & 9 & 14 \\
\hline Spontaneous & 241,355 & 21 & 5 & 14 & 2 & 67 \\
\hline
\end{tabular}

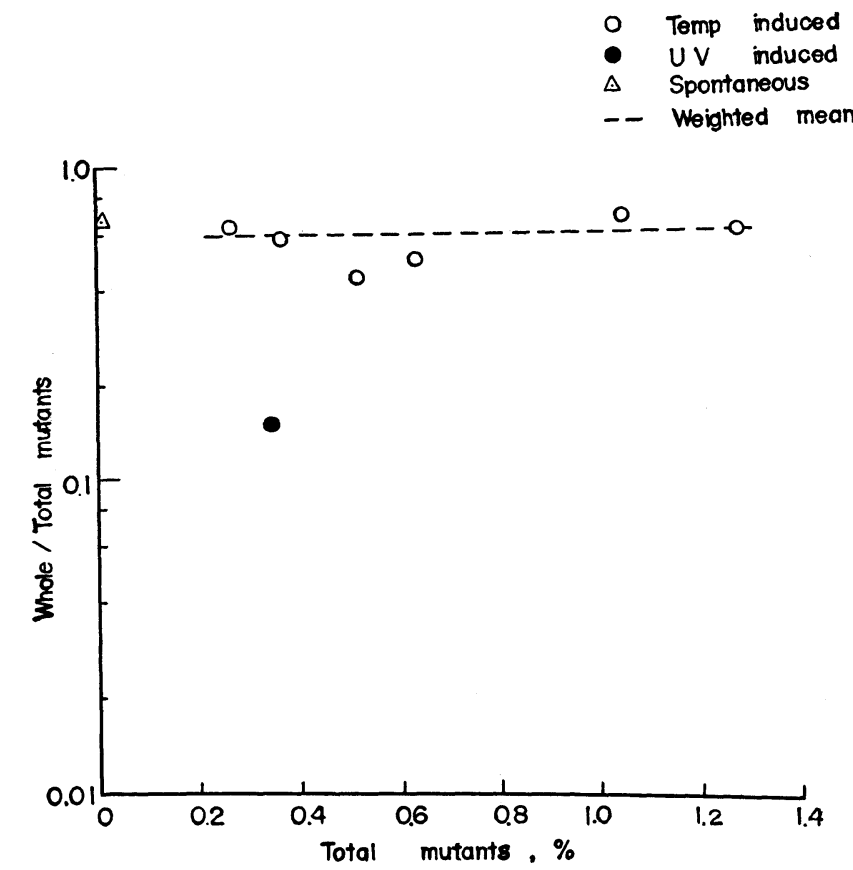

Fig. 3. Fraction of whole-colony mutation (of total mutation) versus total mutation frequency in the high temperature induced and the UV induced.

\section{Discussion}

The action mechanism of high temperature on the DNA molecule can be classified into two types: splitting of the molecule along the axis, and depurination (and to a much lesser extent phosphate diester bond break). The former results in rapid denaturation; whereas the latter is a slow process, and the temperature that leads to this process may be called subcritical temperature (Ginoza and Zimm 1961). In this definition the experimental condition $60^{\circ} \mathrm{C}$ for 4 minutes in this study is apparently subcritical. There is a report on heat resistant strains of $E$. coli that a stable and nuclear mutation was induced at relatively high incidence by subcritical temperature (Zamenhof and Greer 1958). It is also true in yeast cells, as the present report 
indicates. Concerning the mechanism of high temperature action on the DNA molecule, Ginoza and Zimm (1961) were able to show experimentally that inactivation of transforming DNA by subcritical temperature was of the exponential type indicating the process was the one-hit type. Surprisingly enough, in the present study we also encountered the same sort of phenomenon when it was found that whole-colony mutation represented the majority of the total mutation. This may be attributable to a double change in the presumed dual structure of the gene by a single event.

As for the implication of the origin of spontaneous mutation having the same characteristic as temperature induced mutation, it is a recognized relationship in Drosophila (Muller 1955) that the amount of spontaneous mutation accounted by radiation from natural sources is only a fraction of the total spontaneous mutation. In Drosophila (Altenburg and Browning 1961; Schalet 1958) the ratio of whole body mutation to fractionals of spontaneous origin has been reported to be about 50:50, which is fairly close to our result in yeast cells, $67^{3)}: 33$.

A reversible change in the absorption spectrum of cytidylic acid by UV has been reported (Sinsheimer 1957) and its biological significance has been verified by other workers (e. g. Kaplan et al. 1960). Therefore, as a working hypothesis it may be reasonable to assume that in cellular organisms UV would cause a change in one of the two strands of the gene at one time (by a single event). If this is correct, it would produce at sufficiently low dose a colony, a half of which would show mutant character (fractional), and as the dose increases more non-sectored colonies (but of mutant character) would appear because of the increased chance of changes in both strands (by two successive events) during the irradiation. For this reason in order to induce the sectored colony we have to look for such a low dose level that any significant amount of whole-colony mutation (mutation caused by the two events) will be avoided. As the results (Table 1 and Fig. 3) show, we did choose the right one. The case reported by Kaplan et al. (1960) in Chi phage, few mosaic plaques with two-hit type mutation, could be due to too high a dose. It is probably pertinent to point out that with $\mathrm{T}_{4}$ phage (Freese et al. 1961) hydroxylamine (attacks predominantly on cytosine) induced more pure plaque when longer exposure time was employed. An intensive study of the quantitative relationship between dose of UV and fraction of whole-colony mutation has been undertaken to obtain information as to a probability or cross section of the second hit on the opposite strand after the first hit had occurred on the first strand. Preliminary results show that as the dose increases the fraction of whole-colony mutation increases much faster than would be expected by applying the cross section of the first hit to the second hit. This seems to suggest that there is less possibility of the multistructure of the gene being greater than two than of being two. Thus half sectors at very high incidence in the UV induced type may be

3) This value could be an overestimate because of a possible contribution from accumulated mutants through generations previous to the plating. 
taken as evidence of dual nature of information carried by the gene. Less certain in this point are experiments (Kaudewitz 1959; Kaudewitz et al. 1958) done in E. coli because of lack of exact knowledge of the number of nuclei and their ploidy, even though a sector was observed by the use of some mutagenic agents. Another point in this connection is that there is occasionally observable quarter sectors, or less than quarter sectors, or irregular sectors, which pose some problem as to their origin, especially in the UV induced type. They may be delayed mutation, or some other indirect sort of mutation through abnormal metabolic processes; but it is not known anyway whether they involve a double change or a single change or two single changes in the two strands even if the gene has such dual structure. Still another possibility is that the treated cell population is not very homogeneous in terms of multiplicity of gene structure. In other words, in some cells the gene has already had multiplicity of more than two, probably four; while in most cells a multiplicity of two remained at the time of treatment. The extensive study by Altenburg and Browning (1961) with Drosophila sperm has shown that by a chemical mutagenic agent (dimethyl myleran) a mosaic of the half-and-half type is induced in $F_{1}$ males at very high frequency. Fahmy and Fahmy (1961) have reported that mutagenic chemicals with two active groups and one active group have an effect on Drosophila sperm directly related to their respective number of active groups: total body mutant for a chemical with two active groups and mosaic for a chemical with a single active group. Although the action mechanism of the chemicals is not known fully in molecular terms, these results could be favorable evidence for the interpretation developed here.

The somatic crossing-over, proposed by James (James 1955; James and LeeWhiting 1955) as a explanation for the sectored colony with galactose character, may partly explain our present results in the UV treatment but will be failed for some $70 \%$ of the sectored colonies of which the spore test showed that the white isolates were heterozygous. Obviously heterozygous condition can not be appeared by the somatic recombination.

We would like to emphasize that the model we assume in the present paper is far from being proved. It has to be considered as a working model and further experiments have to be designed to prove or disprove it.

\section{Summary}

By using subcritical temperature and ultraviolet light as DNA-attacking agents, the duplex nature of gene mutation of $a d$ locus in yeast cells has been studied at molecular level.

The relative frequencies of whole-colony mutation and fractional-colony (sectored) mutation induced by these two agents are strikingly different: $59 \%$ whole, $23 \%$ fractional and 18\% undefined for subcritical temperature; $14 \%$ whole, $72 \%$ fractional and $13 \%$ undefined for ultraviolet light. The results are interpreted to indicate a 
genetic multiplicity of two of the gene, based on the assumption that subcritical temperature causes changes in both strands of a gene-DNA molecule and ultraviolet in one of the two strands by a single event.

\section{Acknowledgement}

With profound gratitude and respect, we should like to dedicate this paper to the late Professor Koiti Murati, the founder of this Laboratory, without whose kind guidance in his lifetime this work would not have been possible.

We should also like to thank Mr. T. Notake for his technical assistance.

\section{Literature Cited}

Altenburg, E. and L. S. Browning 1961 The relatively high frequency of whole-body mutations compared with fractionals induced by X-rays in Drosophila sperm. Genetics 16: 203-212.

Fahmy, O. G. and M. J. Fahmy 1961 Cytogenetic analysis of the action of carcinogens and tumor inhibitors in Drosophila melanogaster, $\mathrm{X}$. The nature of the mutations induced by the mesyloxy esters in relation to molecular crosslinkage. Genetics 46: 447-458.

Freese, E., E. Bautz-Freese and E. Bautz 1961 Hydroxylamine as a mutagenic and inactivating agent. Jour. Mol. Biol. 3: 133-143.

Ginoza, W. and B. H. Zimm 1961 Mechanism of inactivation of deoxyribonucleic acids by heat. Proc. Nat. Acad. Sci. 47: 639-652.

James, A. P. 1955 A genetic analysis of sectoring in ultraviolet-induced variant colonies of yeast. Genetics 40: 204-213.

and B. Lee-Whiting 1955 Radiation-induced genetic segregations in vegetative cells of diploid yeast. Genetics 40: $826-831$.

Kaplan, R. W., U. Winkler and H. Wolf-Ellmauer 1960 Induction and reversion of c-mutations by irradiation of the extracellular $\chi$-phage of Serratia. Nature 186: 330-331.

Kaudewitz, F. 1959 Production of bacterial mutants with nitrous acid. Nature 183: 1829-1830. , W. von Vielmetter und H. Fréedrich-Freksa 1958 Mutagene Wirkung des Zerfalles von radioaktiven Phosphor nach Einbau in Zellen von Escherichia coli. Zeit. Naturforsch. 13b: 793-802.

Muller, H. J. 1955 How radiation changes the genetic constitution. Bull. of the Atomic Scientists 11: $329-338$.

Sinsheimer, R. L. 1957 The photochemistry of cytidylic acid. Rad. Res. 6: 121-125.

Schalet, A. 1958 A study of spontaneous visible mutations in Drosophila melanogaster. Proc. 10th Int. Congr. Genet. 2: 252.

Zamenhof, S. and S. Greer 1958 Heat as an agent producing high frequency of mutations and unstable genes in Escherichia coli. Nature 182: 611-613. 